\title{
Relationship between Calcification on Mammography and Human Epidermal Growth Factor Receptor 2 (HER-2) Expression in Breast Carcinoma
}

\author{
Zulfadli, ${ }^{1}$ Hari Soekersi, ${ }^{1}$ Raden Yohana, ${ }^{2}$ Birgitta M. Dewayani ${ }^{3}$ \\ ${ }^{1}$ Department of Radiology Faculty of Medicine Universitas Padjadjaran/Dr. Hasan Sadikin General \\ Hospital Bandung, Indonesia, ${ }^{2}$ Department of Internal Medicine Faculty of Medicine Universitas \\ Padjadjaran/Dr. Hasan Sadikin General Hospital Bandung, Indonesia, ${ }^{3}$ Department of \\ Anatomical Pathology Faculty of Medicine Universitas Padjadjaran/Dr. Hasan Sadikin General \\ Hospital Bandung, Indonesia
}

\begin{abstract}
Background: Breast carcinoma shows over-expression of Human Epidermal Growth Factor Receptor 2 (HER-2) in 18 to $20 \%$ of the cases. This type of cancer is very progressive and has a poor prognosis. This study aimed to determine the association between the calcification on mammography with HER-2 expression in breast carcinoma as a marker of breast carcinoma aggressiveness.

Methods: This was an analytic observational study with a case-control design. Patients with breast carcinoma at the Department of Radiology and Department of Anatomical Pathology of Dr. Hasan Sadikin General Hospital Bandung from July-September 2019 were enrolled in this study. Samples were consecutively collected. The association of calcification on mammography and HER2 expression was analyzed using the Chi-square test.

Result: In total, 40 patients were included, consisting of 20 individuals with HER-2 positive and 20 individuals with HER-2 negative. The youngest was 40 years old and the oldest was 73 years old. Statistical test results showed that there was a significant association between calcifications in mammography and HER-2 expression (p-value $=0.0001$, OR 13.22; 95\% CI 2.7-62.6).

Conclusions: There is a significant association between calcification on mammography and HER-2 expression in breast carcinoma, suggesting that positive calcification mammography was 13.22 times higher in patients with HER-2 positive compared to patients with negative calcifications.
\end{abstract}

Keywords: Breast neoplasms, calcification, HER-2, mammography

\section{Introduction}

The first rank of malignancy in women is breast carcinoma. New breast carcinoma cases have been recorded about were 2.1 million in 2018. According to the GLOBOCAN, the mortality rate of breast carcinoma in the world has been predicted as many as 626,000 cases in 2018, and it is a major cause of death in malignant disease. ${ }^{1}$

Mammography is the radiological modality of choice for screening and diagnosis of breast carcinoma, with a sensitivity of $75-85 \%$ and a specificity of $90 \%{ }^{2}$ One mammographic anomaly that is easily detected, and often the earliest signs of malignant breast disease is a very small calcium deposit in breast soft tissue known as micro calcification (MC). Although MC is also associated with benign conditions such as secretory disease and fat necrosis, about $40 \%$ of breast cancer are often accompanied by MC. The MC size is less than $1 \mathrm{~mm}$ and the MC is the only mammographic feature that shows the presence of a tumor and its ppresence is a major risk factor for breast cancer. A previous study has found that MC in malignant lesions tends to be smaller, more numerous, and occur in the milk ducts and other related structures in the breast and follow the anatomy of the duct. ${ }^{3}$ There are several possible causes of calcification, including the development of scar tissue after biopsy or surgery, fluid accumulation, epithelial proliferation, tissue necrosis, and inflammation. Inflammation has been previously linked to

Correspondence: Zulfadli, Department of Radiology Faculty of Medicine Universitas Padjadjaran/Dr. Hasan Sadilkin General Hospital, Jalan Pasteur 38, Bandung, Jawa Barat, Indonesia, Email: fadlipasir@yahoo.com. 
poor breast cancer prognosis and disease progression, possibly due to the recruitment of macrophages that promote tumor growth and proteinases which decrease the extracellular matrix. Suspicious morphology is calcification of mammography that can be suspected in the direction of malignancy can be classified into Breast Imaging Reporting and Data System (BIRADS) $4 \mathrm{~B}$ or $4 \mathrm{C}$ and BI-RADS 5 for fine linear branching calcification. ${ }^{4-6}$

The immunohistochemical (IHC) profile examinations have been used extensively as a basis for selecting hormonal therapy and targeting therapy. Examination of IHC can detect cancer cell hormone receptor types, namely estrogen receptor (ER) and progesterone receptor (PR), as well as expression of human epidermal growth factor receptor-2 (HER-2). HER-2 is a protooncogene that belongs to the epidermal growth factor receptor (EGFR) group. Amplification of HER-2 is considered a poor independent prognostic factor in invasive breast carcinoma and has been associated with changes in clinical response to systemic treatment of breast cancer such as with chemotherapy and antiestrogens. HER-2 is positive in about $18-20 \%$ of breast cancers. Positive HER-2 can be classified as HER-2 type (enriched) if HER2 is positive and estrogenic and progesterone receptors are negative. HER2-positive luminal $B$ if HER-2 is positive and one or both hormones are positive, while negative HER-2 can be classified as luminal A if HER-2 is negative and one or both of the hormone receptors is positive and low Ki-67 and HER2-negative Luminal B if HER-2 is negative and one or both of the hormone receptors is positive and high
Ki- 67 and Basal-like (Triple-negative) if HER2 and both hormone receptors are negative.

Overexpression of HER-2 increases the 'survival' of breast cancer cells by increasing cell proliferation, inhibiting cell apoptosis (death), and increasing angiogenesis by increasing the production of vascular endothelial growth factor. Studies show that there is a correlation between calcifications found on mammography with HER-2 overexpression in primary breast carcinoma patients compared with patients who did not overexpression of HER-2.

Amplification of HER-2 is considered as an independent poor prognostic factor in invasive breast carcinomas and has been associated with altered clinical responsiveness to systemic breast cancer treatment such as chemotherapy and antiestrogens..$^{7-10}$ The aim of the study was to determine the relationship between calcification on mammography and HER-2 expression in breast carcinoma.

\section{Methods}

This study was an observational analytic approach with a case-control study design. Subjects over 40 years old with preoperative breast carcinoma through histopathological examination had undergone immunohistochemical HER-2 examination. The minimum samples number for each group is 20 samples. Then the total sample for the 2 groups is 40 samples divided into 20 subjects with positive HER- 2 results and 20 subjects with negative HER-2 results. All subjects were undergo mammography examination from July to September 2019. This study was approved by the Ethical Committee of Universitas

Table 1 Characteristics of Research Subjects Based on Age and IHC Results

\begin{tabular}{|c|c|c|}
\hline Variable & $n$ & $\%$ \\
\hline \multicolumn{3}{|l|}{ Age (years) } \\
\hline $40-60$ & 33 & 82.5 \\
\hline$>60$ years & 7 & 17.5 \\
\hline \multicolumn{3}{|l|}{ IHC Result } \\
\hline HER-2 type & 12 & 30 \\
\hline HER2-positive Luminal B & 8 & 20 \\
\hline HER2-negative Luminal B & 15 & 37.5 \\
\hline Basal-like /triple- negative & 5 & 12.5 \\
\hline Luminal A & - & - \\
\hline Total & 40 & 100 \\
\hline
\end{tabular}

Note: IHC= immunohistochemistry 


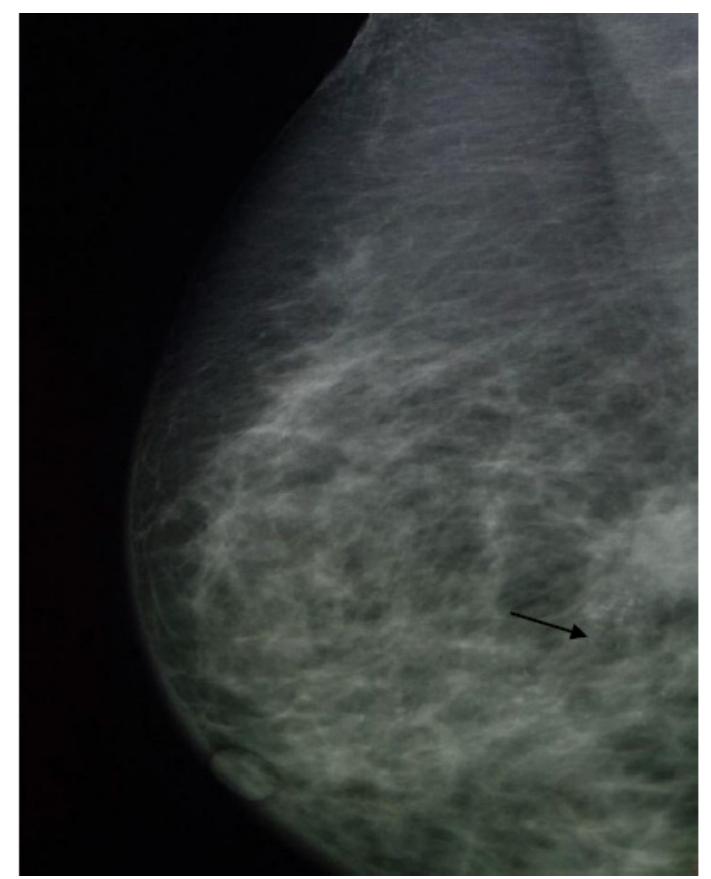

Figure 1 Mammogram (RML0) Showing Microcalcifications (Arrow)

Padjadjaran.

The mammograms were obtained with digital mammography (Metaltronica Helianthus type, Italy). Standard craniocaudal and lateral views were carried out in all subjects. The mammograms of all subjects were blindly reviewed by an experienced breast radiologist. Mammographic findings were categorized as with or without calcifications.

The HER-2 status was determined by IHC staining of tissue sections with primary antibody anti-PD-L1 rabbit monoclonal (clone 28-8, cat No. ab205921, Abcam, Inc Cambridge, USA)with 1:200 dilution and CD133 polyclonal mouse antibody from Elabscience (E-AB-16223) USA with 1:100 dilution. Detection was carried through with streptavidin-biotin immunoperoxide complex. Positive HER-2 if IHC staining +3 (uniform membrane coloring and more than 10\% invasive tumor cells). Negative HER-2 if IHC staining 0 (none membrane coloring and less than $10 \%$ invasive tumor cells) or positive 1 (weak membrane coloring and more than $10 \%$ invasive tumor cells). The samples analysis was done by a pathologist that was an expert in IHC examination at the Department of Anatomical Pathology Dr. Hasan Sadikin General Hospital.

The Chi-square test was used to evaluate the association between mammographic findings and HER-2 expression in breast carcinomas. All statistical tests were twosided while the statistical significance of the observed difference was set at $\mathrm{p}<0.05$. The Odds ratio (OR) value resulted from the Chisquare test was to measure the strength of causal and effect relationships. All data were analyzed using Statistical Package for the Social Sciences statistical software (SPSS) version 24.0 for windows.

\section{Results}

Most breast cancer patients were aged 40-60 years $(82.5 \%)$, and most with HER-2 negative luminal B IHC results (37.5\%). Characteristics of research subjects based on age and IHC results in study subjects were presented in Table 1).

The mammoghrapy result with MC from a patient was shown in Figure 1. Characteristics of the study subjects based on the findings of calcifications in each molecular subtype were depicted in Figure 2. The relationship of calcification on mammography with HER-2 overexpression compared with patients who did not overexpress HER-2 in breast carcinoma sufferers was shown in Table 2.

The relationship of calcification on mammography with HER-2 overexpression compared with patients who did not in breast carcinoma sufferers. In the positive HER-2 


\section{CALCIFICATION IN MOLECULAR} SUBTYPES

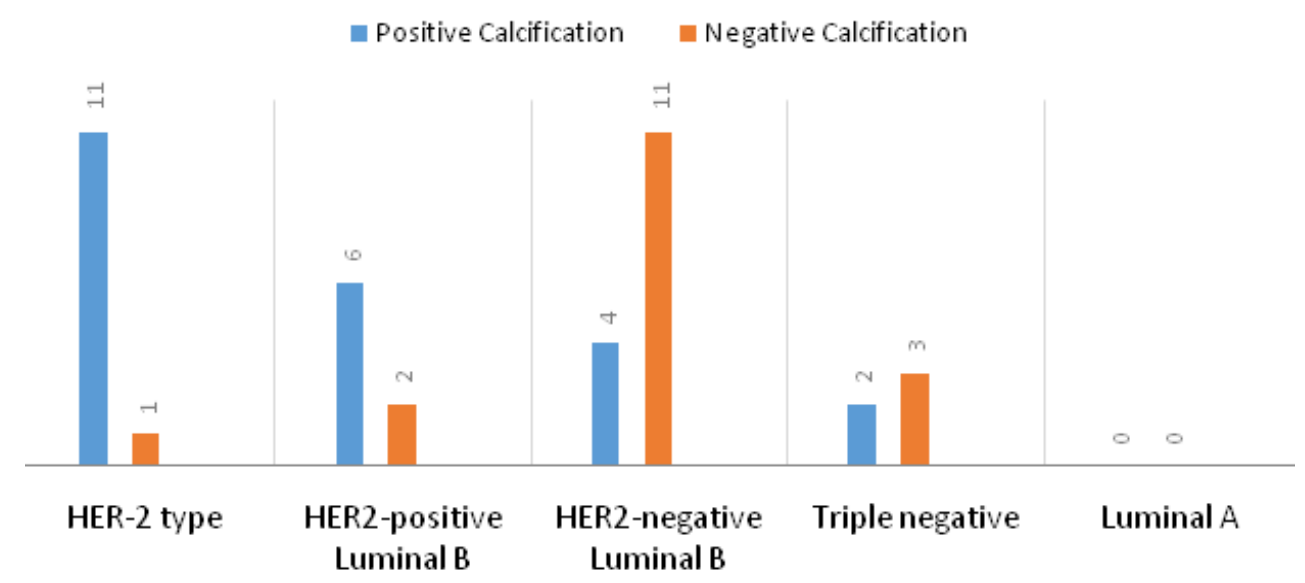
Figure 2 Characteristics of Study Subjects based on the Findings of Calcification in Each
Molecular Subtype

group, patients with positive calcification mammography were $17(85.0 \%)$ and negative calcifications were $3(15.0 \%)$. In the negative HER-2 group, patients with positive calcification mammography were $6(30.0 \%)$ and negative calcifications were 14 (70.0\%).

Chi-Square test analysis results obtained a $\mathrm{p}$-value of 0.0001 , meaning that there was a statistically significant relationship between calcification in mammography with HER2 expression in breast carcinoma. The OR was 13.22 (95\% CI 2.7-62.6), suggesting that positive calcification mammography was 13.22 times higher in positive of HER-2 compared to patients with negative calcifications.

\section{Discussion}

Our study shows that the most age distribution with carcinoma in the breast was the age group 40-60 years (82\%). This is following the literature that the incidence of breast cancer increases with age and 95\% of cases of breast cancer occur in women aged 40 years or more. Breast cancer rarely occurs at a young age of less than 40 years. ${ }^{11}$ The most IHC results from this study subjects are HER-2 negative luminal B (37\%). It was similar to another study that reported the highest incidence is HER-2 negative luminal B $(31 \%) .^{12}$ In a prospective study has found that triple negative as the most subtypes (23\%) followed by HER-2 negative luminal B (21\%). ${ }^{10}$

Interestingly, positive calcifications have been shown in $15 \%$ of subjects with negative HER-2 similar to other research $(10 \%)^{10}$ Calcification in subjects with negative HER-2 is thought to be related to factors other than HER-2 such as hormonal factors. Another study has found a relationship between hormonal

Table 2 Relationship of Calcification on Mammography with HER-2 Overexpression Compared with Did Not Overexpress HER-2 in Patients with Breast Carcinoma.

\begin{tabular}{|c|c|c|c|c|}
\hline \multirow{3}{*}{ Mammography } & \multicolumn{2}{|c|}{ Group } & \multirow{3}{*}{$\begin{array}{c}\text { OR } \\
\text { CI (95\%) }\end{array}$} & \multirow{3}{*}{ P-value } \\
\hline & HER-2 Positive & HER-2 Negative & & \\
\hline & $\mathrm{n}=\mathbf{2 0}$ & $\mathrm{n}=\mathbf{2 0}$ & & \\
\hline Positive calcification & $17(85.0 \%)$ & $6(30.0 \%)$ & 13.22 & 0.0001 \\
\hline Negative calcification & $3(15.0 \%)$ & $14(70.0 \%)$ & $(2.790-62.670)$ & \\
\hline
\end{tabular}

Note: Chi -Square test*. Significance value base on $p$-value $<0.05$ 
receptors which are positive estrogen receptor and calcification on mammography. ${ }^{6}$ Calcification may have another cause such as a history of inflammation and previous tissue necrosis. ${ }^{6}$ Positive calcifications in the HER-2 negative luminal $B$ and triple negative molecular subtypes which are HER-2 negative groups may give negative calcification imaging on mammography, with HER-2 negative luminal $B$ as the most calcifications in the negative HER-2 group. Another study has shown that luminal A, HER-2 negative luminal $B$ and triple negative in the negative HER-2 group which provided calcification on mammography with luminal $\mathrm{A}$ as the most subtypes. ${ }^{10}$

Negative calcifications in the positive HER2 group (15\%) may occur in positive HER-2 luminal B as also found in another study that showed samples in the positive HER-2 group with negative MC on mammography $(20 \%){ }^{10}$ Figure 2 shows that the most MC have been found in the HER-2 type, similar to another study that the most calcifications in the HER2 type subtype have positive calcifications. , $^{6,10}$

Table 2 has shown a significant relationship between calcification on mammography and HER-2 expression in breast carcinoma. In this study, positive calcifications found (85\%) are associated with positive HER-2. Another study has shown positive calcifications and positive of HER-2 expression in more than $60 \%$.,10 The correlation of calcifications on mammography with HER-2 expression with an OR of 13.22, suggesting that the risk of positive HER-2 on positive calcification on mammography is 13.22 times compared to negative calcification. Another study shows a relationship with a higher OR of 8.1.10

The weakness of the study is that there are subjects who still have dense fibrogranular tissue, although they are over 40 years old. Therefore, the position on mammography needs to be improved. Also, some subjects have large and hard masses, making it difficult to cover by mammography tools.

In conclusion, there is a significant relationship between calcification on mammography with HER-2 overexpression in breast carcinoma with an OR of 13.22. Calcifications detected during mammography not only have diagnostic value but it can also predict the choice of therapy.

\section{References}

1. Bray F, Ferlay J, Soerjomataram I, Siegel RL, Torre LA, Jemal A. Global cancer statistics 2018: GLOBOCAN estimates of incidence and mortality worldwide for 36 cancers in 185 countries. CA Cancer J Clin. 2018;68(6):394-424.

2. Tadwalkar RV, Rapelyea JA, Torrente J, Rechtman LR, Teal CB, McSwain AP, et al. Breast-specific gamma imaging as an modality for the diagnosis of invasive breast cancer with correlation to tumor size and grade. Br J Radiol. 2012;85(1014):e212-6.

3. Cai H, Huang Q, Rong W, Song Y, Li J, Wang J, et al. Breast microcalcification diagnosis using deep convolutional neural network from digital mammograms. Comput Math Methods Med. 2019;2019:2717454.

4. Naseem M, Murray J, Hilton JF, Karamchandani J, Muradali D, Faragalla H, et al. Mammographic microcalcifications and breast cancer tumorigenesis: A radiologic-pathologic analysis. BMC Cancer. 2015;15:307.

5. Zonderland $\mathrm{H}$, Smithuis R. Bi-RADS for mammography and ultrasound 2013. Netherlands: Radiology Assistant; 2014 [cited 2020 January 20] Available from https://radiologyassistant.nl/ breast/bi-rads-for-mammography-andultrasound-2013

6. Nyante SJ, Lee SS, Benefield TS, Hoots TN, Henderson LM. The association between mammographic calcifications and breast cancer prognostic factors in a population-based registry cohort. Cancer. 2017:123(2):219-27.

7. Wiguna NIP, Manuaba IBTW. Karakteristik pemeriksaan imunohistokimia pada pasien kanker payudara di RSUP Sanglah periode 2003-2012. E-Jurnal Medika Udayana. 2012;3(7). [cited 2020 January 20] Available from: https://ojs.unud.ac.id/ index.php/eum/article/view/9809

8. Elias SG, Adams A, Wisner DJ, Esserman LJ, Van't Veer LJ, Mali WPThM, et al. Imaging features of HER2 Overexpression in Breast Cancer: A systematic Review and Metaanalysis. Cancer Epidemiol Biomarkers Prev. 2014;23(8):1464-83.

9. Wang $\mathrm{X}$, Chao $\mathrm{L}$, Chen $\mathrm{L}$, Tian $\mathrm{B}$, Ma G, Zang Y, et al. Correlation of mammographic calcifications with HER$2 /$ neu overexpression in primary breast carcinomas. J Digit Imaging. 2008;21(2): 170-6.

10. Rashmi S, Kamala S, Murhty SS, Kotha S, Rao YS, Chaudhary KV. Predicting the molecular subtype of breast cancer based on mammography and ultrasound findings. Indian J Radiol Imaging. 2018;28(3):354-61. 
11. Jardines L, Goyal S, Fisher P, Royce M Weitzel J, Royce M, Goldfarb SB. Breast cancer overview: risk factors, screening, genetic testing, and prevention. Cancer network. 2015 [cited 2020 January 20] Available from: https://www. cancernetwork.com/view/breast-canceroverview-risk-factors-screening-genetic- testing-and-prevention

12. Kondov B, Milenkovikj Z, Kondov G, Petrushevska G, Basheska N, BogdanovskaTodorovska M, et al. Presentation of the molecular subtypes of breast cancer detected by immunohistochemistry in surgically treated patients. Open Access Maced J Med Sci. 2018;6(6):961-7. 